\title{
Deciding Reachability for Piecewise Constant Derivative Systems on Orientable Manifolds
}

\author{
Andrei Sandler and Olga Tveretina \\ Department of Computer Science, \\ University of Hertfordshire, \\ United Kingdom \\ $\{$ a.sandler, o.tveretina $\}$ @herts.ac.uk
}

\begin{abstract}
A hybrid automaton is a finite state machine combined with some $k$ real-valued continuous variables, where $k$ determines the number of the automaton dimensions. This formalism is widely used for modelling safety-critical systems, and verification tasks for such systems can often be expressed as the reachability problem for hybrid automata.

Asarin, Mysore, Pnueli and Schneider defined classes of hybrid automata lying on the boundary between decidability and undecidability in their seminal paper 'Low dimensional hybrid systems - decidable, undecidable, don't know' [9]. They proved that certain decidable classes become undecidable when given a little additional computational power, and showed that the reachability question remains unsolved for some 2-dimensional systems.

Piecewise Constant Derivative Systems on 2-dimensional manifolds (or $\mathrm{PCD}_{2 m}$ ) constitute a class of hybrid automata for which decidability of the reachability problem is unknown. In this paper we show that the reachability problem becomes decidable for $\mathrm{PCD}_{2 m}$ if we slightly limit their dynamics, and thus we partially answer the open question of Asarin, Mysore, Pnueli and Schneider posed in [9].
\end{abstract}

Keywords: Hybrid Systems · Reachability · Decidability

\section{Introduction}

A hybrid automaton is a formalism used to model dynamic systems that comprise both digital and analog components. Formally, it is a finite state machine combined with some $k$ real-valued continuous variables, where $k$ determines the number of the automaton dimensions. Examples of such systems can be found among others in avionics, robotics and bioinformatics, and most of them are safety-critical.

Verifying safety properties typically consists of construction of a set of reachable states and checking whether this set intersects with a set of unsafe states. Therefore, one of the most fundamental problems in the analysis of hybrid automata is the reachability problem. Formally, it is stated as follows: for a given automaton determine if there is a trajectory from some initial state to a target state. 
Undecidability of reachability is usually proved by the simulation of a Turing machine or any other Turing-complete abstraction on the given hybrid automaton (see $[9,3]$ for examples). This way, the existence of an algorithm deciding the reachability problem would solve the halting problem, which is a contradiction. On the other hand, decidability of reachability is typically shown by providing an algorithm which solves it, or by showing that the system admits finite bisimulation $[13,14]$.

The reachability problem is undecidable even for simple classes of hybrid automata such as linear hybrid automata [1]. Nevertheless, there are classes of hybrid systems for which it is decidable. Examples of decidable systems include o-minimal systems [13] and initialized rectangular automata [11].

Despite the increasing interest in discovering new decidability results for hybrid automata, there is still no clear boundary between what is decidable and what is not for such systems [9].

Asarin et al. and Henzinger et al. presented hybrid automata that span the boundary between decidability and undecidability for the reachability problem in [9] and [11] respectively. Asarin et al. observed that certain decidable classes become undecidable, when given a little additional computational power. Thus, decidable 2-dimensional Piecewise Constant Derivative Systems (PCDs) become undecidable for three dimensions or higher [3, 15].

Asarin and Schneider considered 2-dimensional Hierarchical Piecewise Constant Derivative Systems (HPCDs), an intermediate class lying between decidable 2-dimensional and undecidable 3-dimensional PCDs [4]. They proved that 2-dimensional HPCDs are equivalent to 1-dimensional Piecewise Affine Maps (PAMs), a class of dynamical systems for which reachability is a well-known open problem [8]. A 1-dimensional PAM is a piecewise function which is applied to the 1-dimensional real line, and the function within each interval of the real line is affine. They are proven to be equivalent to a 2-dimensional system called a planar pseudo-billiard system, also known as a 'strange billiards' model in bifurcation and chaos theory [12].

Variants of HPCDs called Restricted HPCDs (RHPCDs), have been considered in [7]. This class of systems has similarities with many well-known models such as rectangular automata and stopwatch automata. The authors show that 3-dimensional RHPCDs are undecidable by encoding a Minsky machine.

Mysore and Pnueli raised the following question [17]: Is there any class, simpler than 2-dimensional HPCDs, which is equivalent to 1-dimensional PAMs? Asarin et al. came up with further classes of hybrid automata, including PCDs on 2-dimensional manifolds $\left(\mathrm{PCD}_{2 m}\right)$, which are equivalent to 1-dimensional PAMs [9].

We consider $\mathrm{PCD}_{2 m}$ with slightly limited dynamics by forbidding colliding and branching trajectories. We call such systems Regular $\mathrm{PCD}_{2 m}\left(\mathrm{PCD}_{r 2 m}\right)$ and show that the reachability problem is decidable in this case.

As 'reference systems' we use dynamical systems on the closed orientable surfaces and rely on the topological properties of their trajectories [16]. Furthermore, we study the properties of the language generated by the trajectories of 
a $\mathrm{PCD}_{r 2 m}$ by associating all generated words with a sequence of graphs, called Rauzy graphs or factor graphs [18].

The remainder of the paper is organised as follows. In Section 2 we recall the notion of 2-dimensional PCDs, and in Section 3 we extend it to manifolds. Section 4 provides properties of dynamical systems on the closed orientable surfaces.

In Section 5 we introduce the language of $\mathrm{PCD}_{r 2 m}$, and in Section 6 we show that the reachability problem can be solved for $\mathrm{PCD}_{r 2 m}$ is decidable. Section 7 contains concluding remarks.

\section{Preliminaries}

A Piecewise Constant Derivative System (PCD) can be viewed as a finite set of regions, where each region is associated with a vector field which determines the rate of change of the continuous variables. In this section we formally define them including the reachability problem for such systems.

\subsection{Piecewise Constant Derivative Systems on a Plane}

In this paper we deal with a 2-dimensional Euclidean space $X=\mathbb{R}^{2}$. An open half-space in $X$ is the set of all points $\boldsymbol{x} \in X$ satisfying $\boldsymbol{a} \cdot \boldsymbol{x}+b<0$ for some rational $\boldsymbol{a}$ and $b$. A convex open polygonal set $q$ is an intersection of a finite number of half-spaces, and $\mathrm{cl}(q)$ denotes the closure of $q$.

A finite polygonal partition of $X$ is a set $\mathrm{Q}=\left\{q_{1}, \ldots, q_{k}\right\}$ of polygonal sets, called regions, such that: (1) $q_{i} \neq \varnothing$ for all $1 \leq i \leq k ;(2) q_{i} \cap q_{j}=\varnothing$ for all $1 \leq i, j \leq k$ such that $i \neq j ;(3) \bigcup_{i=1}^{k} \operatorname{cl}\left(q_{i}\right)=X$

The boundary of each region $q \in \mathrm{Q}$ is $\operatorname{bd}(q)=\mathrm{cl}(q) \backslash q$. The interior $\operatorname{int}\left(X^{\prime}\right)$ of $X^{\prime} \subseteq X$ is the set of points $\boldsymbol{x} \in X^{\prime}$ such that for some $\varepsilon>0$ there exists an $\varepsilon$-neighbourhood $N_{\varepsilon}(\boldsymbol{x}) \subseteq X^{\prime}$ of $\boldsymbol{x}$. If $X^{\prime}$ is 1-dimensional then $\varepsilon$-neighbourhood is assumed 1-dimensional too.

We use $\mathrm{E}(\mathrm{Q})$ to denote the set of edges of $\mathrm{Q}$ of the form $e=\operatorname{int}\left(\operatorname{cl}\left(q_{i}\right) \cap \mathrm{cl}\left(q_{j}\right)\right)$, where $q_{i}, q_{j} \in \mathrm{Q}, i \neq j$, and $\operatorname{int}\left(\mathrm{cl}\left(q_{i}\right) \cap \mathrm{cl}\left(q_{j}\right)\right) \neq \varnothing$. Similarly, $\mathrm{V}(\mathrm{Q})$ denotes the set of vertices of $\mathrm{Q}$ of the form $v=\operatorname{cl}\left(e_{i}\right) \cap \mathrm{cl}\left(e_{j}\right)$, where $e_{i}, e_{j} \in \mathrm{E}(\mathrm{Q}), i \neq j$, and $\mathrm{cl}\left(e_{i}\right) \cap \mathrm{cl}\left(e_{j}\right) \neq \varnothing$.

We say that $B d(Q)=E(Q) \cup V(Q)$ is a set of border elements. Now the set $\mathrm{Q} \cup \mathrm{Bd}(\mathrm{Q})$ forms a partition of $X$. We define the border elements of a region $q \in \mathrm{Q}$ as $\operatorname{Bd}(q)=\{b \mid b \subseteq \mathrm{cl}(q)\} \cap \operatorname{Bd}(\mathrm{Q})$.

Definition 1 (2-PCD). A 2-dimensional Piecewise Constant Derivative System (or 2- $P C D$ ) is a pair $\mathrm{H}=(\mathrm{Q}, \mathrm{F})$ with $\mathrm{Q}=\left\{q_{i}\right\}_{i \in I}$ a finite polygonal partition of $\mathbb{R}^{2}$ and $\mathrm{F}=\left\{\boldsymbol{v}_{i}\right\}_{i \in I}$ a set of vectors from $\mathbb{R}^{2}$. The dynamics is determined by the equation $\dot{x}=\boldsymbol{v}_{i}$ for $x \in q_{i}$.

We can also define a 2-PCD on a convex subset $S \subset X$ and assume that the dynamics on the rest of $\mathbb{R}^{2}$ is defined as a constant flow going in or out from our 2-PCD, depending on the flow of the boundary of $S$.

The set $\operatorname{Bd}(q)$ consists of all boundary elements of a region $q \in \mathrm{Q}$ - edges and vertices. Now we define the input and output boundary elements of $q$. 


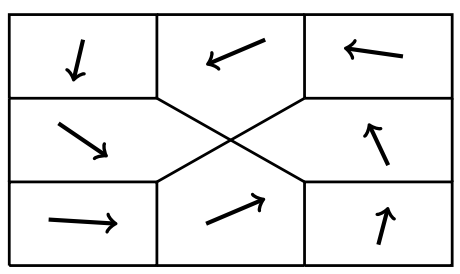

(a)

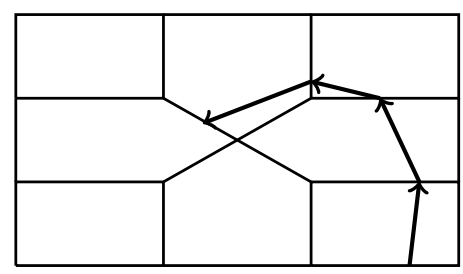

(b)

Fig. 1. (a) An example of a 2-PCD; (b) an example of a trajectory segment.

Definition 2 (Input and output edges). Assume $q \in \mathrm{Q}$ with dynamics $\boldsymbol{v}$, and an edge $e \in \operatorname{Bd}(q)$. We say that $e$ in an input edge for $q$ if for any $x \in e$ there is $t>0$ such that $x+\boldsymbol{v} t \in q$; and $e$ in an output edge for $q$ if for any $x \in e$ there is $t<0$ such that $x+\boldsymbol{v} t \in q$.

By Definition 1, for every vertex $x \in \operatorname{bd}(q)$ with $q \in \mathrm{Q}$ there are exactly two edges $e, e^{\prime} \subseteq \mathrm{bd}(q)$ such that $x \in \mathrm{cl}(e) \cap \mathrm{cl}\left(e^{\prime}\right)$.

Definition 3 (Input and output vertices). We say that $x$ is an input vertex for $q$ if both $e$ and $e^{\prime}$ are input edges; $x$ is an output vertex for $q$ if both $e$ and $e^{\prime}$ are output edges; and $x$ is neutral with respect to $q$ if $e$ is an input edge and $e^{\prime}$ is an output edge.

We denote by $\ln (q) \subseteq \mathrm{Bd}(q)$ and $\operatorname{Out}(q) \subseteq \mathrm{Bd}(q)$ the sets of input and output border elements (edges and vertices) of some region $q$ respectively. In the rest of the paper and similar to [5] we assume that $\ln (q) \cap \operatorname{Out}(q)=\varnothing$.

\section{$2.2 \quad$ Trajectories}

In this section we define the notions of a trajectory, its discrete abstraction called an edge signature, and successor functions similar to $[5,6]^{1}$.

Definition 4 (Trajectory). A trajectory segment of $\mathrm{H}=(\mathrm{Q}, \mathrm{F})$ with the starting point $\boldsymbol{x}_{0}$ is a continuous and almost-everywhere (except on finitely many points) derivable function $\tau:[0, T] \rightarrow \mathbb{R}^{2}$ such that $\tau(0)=\boldsymbol{x}_{0}$ and for any $t \in[0, T]$, if $\tau(t) \in q_{i}$ then $\dot{\tau}(t)=\boldsymbol{v}_{i}$. If $T=\infty$ then $\tau$ is called a trajectory.

In the following we consider the discrete abstraction of a trajectory called an edge signature.

Definition 5 (Edge signature). The edge signature of a trajectory $\tau$ is the sequence $\sigma(\tau)=e_{0} e_{1} e_{2} \ldots$ of edges traversed by $\tau$.

\footnotetext{
${ }^{1}$ A PCD can be seen as a special case of polygonal differential inclusion systems.
} 
The edge signature of any trajectory segment $\tau$ can be represented in the following form:

$$
\operatorname{Sig}(\tau)=r_{1} s_{1}^{k_{1}} r_{2} s_{2}^{k_{2}} \ldots r_{n} s_{n}^{k_{n}} r_{n+1}
$$

where $s_{i}^{k_{i}}$ denotes the cycles $s_{i}$ of edges repeated $k_{i}$ times, and $r_{i}$ denotes the paths (sequence of edges) between cycles (see Theorem 4.1 in [6]). Cycles $s_{i}$ are simple, that is, an edge can not appear twice in the cycle.

Definition 6 (Signature type). The signature type of an edge signature $\operatorname{Sig}(\tau)=r_{1} s_{1}^{k_{1}} r_{2} \ldots r_{n} s_{n}^{k_{n}} r_{n+1}$ is the sequence type $(\tau)=r_{1} s_{1} r_{2} \ldots r_{n} s_{n} r_{n+1}$.

The following theorem defines the set of signature types which has to be examined to compute reachable states for a given 2-PCD.

Theorem 1 (Asarin, Schneider, Yovine, [6]). Only those signature types having disjoint paths $r_{i}$ and unique (as sets of edges) cycles $s_{i}$, could correspond to a trajectory starting in initial set $S$ and ending in final set $F$. There are only finite number of such signature types on any given 2-PCD.

For computing the successive interval images, it is convenient to introduce a one-dimensional coordinate system on each edge $e$, with zero (0) denoting one chosen vertex $v_{0}$ of $e$ and one (1) denoting the other vertex $v_{1}$. Now each point has the coordinate $v_{\lambda}=\lambda v_{0}+(1-\lambda) v_{1}$ with $0<\lambda<1$. Then, a series of successor functions on edges of the 2-PCD can be defined.

- Let $x \in e$ for some $e \in \operatorname{In}(q)$. The $\operatorname{successor} \operatorname{Succ}(x, q)$ of $x$ is a point $x^{\prime} \in e^{\prime}$ for some $e^{\prime} \in \operatorname{Out}(q)$ such that there is a trajectory segment that starts in $x$, ends in $x^{\prime}$ and goes only through $q$.

- Let $\left(x_{1}, x_{2}\right) \subseteq e$ for some $e \in \ln (q)$. The successor Succlnt $\left(x_{1}, x_{2}, e^{\prime}, q\right)$ of $\left(x_{1}, x_{2}\right)$ on the interval $e^{\prime} \in \operatorname{Out}(q)$ is $\left(x_{1}^{\prime}, x_{2}^{\prime}\right) \subseteq e^{\prime}$ defined as follows:

$$
x_{1}^{\prime}=\min \left(1, \operatorname{Succ}\left(x_{1}, q\right), x_{2}^{\prime}=\max \left(0, \operatorname{Succ}\left(x_{2}, q\right)\right)\right.
$$

If $x_{1}^{\prime}>x_{2}^{\prime}$, then the interval successor is the empty set. In other words, the successor of an interval $\left(x_{1}, x_{2}\right)$ of an input edge $e$ is the maximal interval $\left(x_{1}^{\prime}, x_{2}^{\prime}\right)$ of an output edge $e^{\prime}$ reachable under the region's dynamics.

\subsection{Reachability problem}

Reachability for PCD-like systems can typically be formulated as either point-topoint or edge-to-edge reachability. In this paper we are interested in edge-to-edge reachability.

Definition 7 (Point-to-point reachability). Let $\mathrm{H}=(\mathrm{Q}, \mathrm{F})$ be a $P C D$. Then a point $b \in \mathrm{Bd}(\mathrm{Q})$ is reachable from a point $a \in \mathrm{Bd}(\mathrm{Q})$ if there is a trajectory segment that starts at $a$ and ends at $b$.

Definition 8 (Edge-to-edge reachability). Let $\mathrm{H}=(\mathrm{Q}, \mathrm{F})$ be a $P C D$. Then an edge $e_{f} \in \mathrm{E}(\mathrm{Q})$ is reachable from an edge $e_{s} \in \mathrm{E}(\mathrm{Q})$ if there are points $a_{s} \in e_{s}$ and $b_{f} \in e_{f}$ such that there is a trajectory segment that starts at $a_{s}$ and ends at $b_{f}$. 


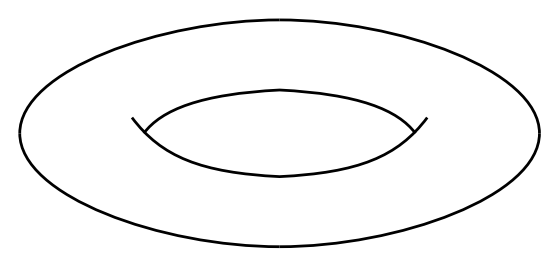

(a)

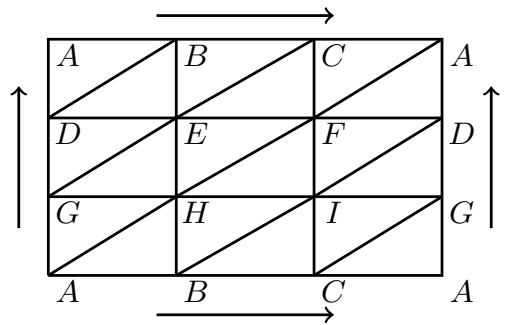

(b)

Fig. 2. Different representations of a torus: (a) A surface in $\mathbb{R}^{3}$; (b) A triangulated surface with identified edges.

\section{Piecewise Constant Derivative Systems on Manifolds}

All the definitions in this section are similar to the respective definitions in [9] and follow the combinatorial approach in [10].

Definition 9 (Triangulable space). A topological space is triangulable if it is obtained from a set of triangles by the identification of edges and vertices, where any two triangles are identified either along a single edge or at a vertex, or are completely disjoint. The identification is done via an affine bijection.

By a closed surface we mean a compact surface without boundary, and it is formally defined below.

Definition 10 (Closed surface). A closed surface (or a 2-dimensional manifold) $S$ is a compact triangulable space for which in addition the following holds:

(1) Each edge is identified with exactly one other edge;

(2) The triangles identified at each vertex can always be arranged in a cycle $T_{1}, T_{2}, \ldots, T_{k}, T_{1}$ so that adjacent triangles are identified along an edge.

Examples of closed surfaces include a sphere, a torus (see Figure 2) and projective planes. In the rest of the paper we only deal with orientable surfaces (a sphere and a connected sum of tori) even though we do not always state it explicitly.

Definition $11\left(\mathbf{P C D}_{2 m}\right)$. We define a $P C D$ on a 2-dimensional manifold (or $\left.P C D_{2 m}\right) \mathrm{H}=(\mathrm{Q}, \mathrm{F})$ as a 2-PCD on a closed orientable surface $S$.

Below we introduce the subclass of $\mathrm{PCD}_{2 m}$ which we will prove to be decidable. We define a Regular $\mathrm{PCD}_{2 m}$ by imposing additional restrictions on the dynamics (the flow vectors do not collide or diverge on edges and vertices) to guarantee that any point of the trajectory has exactly one predecessor and one successor. 


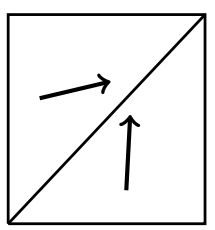

(a)

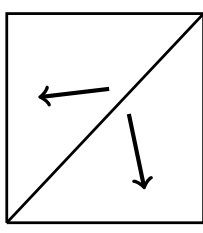

(b)

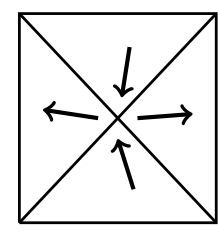

(c)

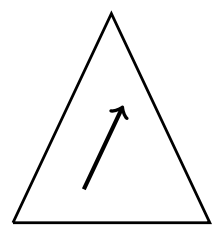

(d)

Fig. 3. Forbidden dynamics in $\mathrm{PCD}_{r 2 m}$ : (a) collision on an edge; (b) branching on an edge; (c) collision and branching on a vertex; (d) flow vector parallel to an edge.

Definition $12\left(\mathbf{P C D}_{r 2 m}\right)$. We say that a $P C D_{2 m} \mathrm{H}=(\mathrm{Q}, \mathrm{F})$ is regular (or $\left.P C D_{r 2 m}\right)$ if the following holds:

1. For any $q, q^{\prime} \in \mathrm{Q}$, if $b \in \operatorname{Bd}(q) \cap \operatorname{Bd}\left(q^{\prime}\right)$ then either $b \in \ln (q)$ and $b \in \operatorname{Out}\left(q^{\prime}\right)$, or $b \in \operatorname{Out}(q)$ and $b \in \ln \left(q^{\prime}\right)$.

2. The vector field in any $q \in \mathrm{Q}$ is not parallel to any $e \in \mathrm{cl}(q) \cap \mathrm{E}(\mathrm{Q})$.

Examples of dynamics forbidden for $\mathrm{PCD}_{r 2 m}$ by Definition 12 are given in Figure 3. Now a manifold is a finite set of triangles with identified edges, and the constant vector field in each triangle defines a successor relation between edges of the triangle such that each triangle has either two input edges and one output edge or one input and two output edges.

\section{Dynamical Systems on the Closed Orientable Surfaces}

Dynamical systems on the closed orientable surfaces and topological properties of their trajectories considered in [16] provide a formalism promising to serve as a 'reference system' for showing decidability of reachability problem on $\mathrm{PCD}_{r 2 m}$.

Let $S_{g}$ be a closed orientable 2-dimensional manifold (surface) with the genus $g \geqslant 0$, and $R$ be a covering of this manifold by a finite number of regions $r_{i}$, $1 \leqslant i \leqslant n$, homeomorphic to a Euclidean disc such that every region $r_{i}$ has its own coordinate system $\left(\varphi_{i}, \psi_{i}\right)$. Let the dynamics in each $r_{i}$ be defined by a system of differential equations:

$$
\varphi_{i}^{\prime}=\Phi\left(\varphi_{i}, \psi_{i}\right), \quad \psi_{i}^{\prime}=\Psi\left(\varphi_{i}, \psi_{i}\right)
$$

Furthermore, we assume the following:

- Transformation of one coordinate system to another at the points which belong to two regions or more is done by continuous functions with continuous derivatives and nonzero Jacobian;

- The right sides of the Equation 1 are continuous functions and become zero only at a finite number of points;

- Dynamics change between regions is done by the functions transforming one coordinate system to another. 
For convenience we refer such systems as Regionwise Dynamical Systems on 2-dimensional manifolds $\left(\mathrm{RDS}_{2 m}\right)$ in the rest of the paper.

In this study we are concerned with three different types of trajectories. Two of them, dense and orbital stable trajectories are defined below. The third type of interest are trajectories on a subset of $\mathbb{R}^{2}$ and their properties are described in Section 2.

Definition 13 (Dense trajectory). A trajectory $\tau$ is dense on a set of intervals $e_{1}, \ldots, e_{k}$ if for any $x \in \tau$ and any interval $e_{i}^{\prime} \subseteq e_{i}, 1 \leqslant i \leqslant k$, there is $y \in e_{i}^{\prime}$ such that $y$ is reachable from $x$.

Definition 14 (Orbital stable trajectory). A trajectory $\tau$ is called orbital stable if for any $\varepsilon>0$ there exists $\delta>0$ such that if a trajectory $\tau^{\prime}$ starts in the $\delta$-neighbourhood of $\tau$ then it is also contained in the $\varepsilon$-neighbourhood of $\tau$.

Along with the covering of the given manifold by the regions as described above, we also consider another covering. Theorem 2 below shows that any $\mathrm{RDS}_{2 m}$ can be decomposed into components consisting of trajectories which are equivalent topologically.

Theorem 2 (Mayer, [16]). A $R D S_{2 m} S_{g}$ is a disjoint union of a finite number of areas $M_{1}, \ldots, M_{k}$ (referred later as dynamical components) of the following types:

1. Type A: Any trajectory inside the area is orbital stable and non-closed. Furthermore, all the trajectories have the same set of limit points; the area is flat and at most 2-connected;

2. Type B: Any trajectory inside the area is closed; the area is either flat and at most 2-connected or equals to the whole manifold in case of $g=1$ (only for a torus);

3. Type C: Any trajectory inside the area is everywhere dense; the area is not flat and the number of areas of this type does not exceed $g$.

All other trajectories, called separatrices, form boundaries between the areas of the above types.

Proposition 1. Any $P C D_{r 2 m}$ is a $R D S_{2 m}$, and therefore it is a disjoint union of a finite number of areas of types $A, B$ and $C$ as defined in Theorem 2 and separatrices.

Proof. By definition of $\mathrm{PCD}_{r 2 m}$ the dynamics change between regions is nondegenerate and local coordinates change with nonzero Jacobian. The flow in each region is constant, hence there is a local coordinate system where both $\varphi_{i}^{\prime}$ and $\psi_{i}^{\prime}$ are nonzero.

Proposition 2. Any separatrix of $P C D_{r 2 m} \mathrm{H}=(\mathrm{Q}, \mathrm{F})$ starts and ends at a vertex. 
Proof. Let us assume that there is an infinite (or half-infinite) separatrix $\tau_{s}$. Then there is an $\varepsilon$-tube around $\tau_{s}$ (see Figure 4) such that the trajectories from different sides of $\tau_{s}$ belong to different dynamic components but never split, which contradicts to the definition of a separatrix (a trajectory separating areas of different types).

Note that there could be trajectories that starts and ends in vertices that are not separatrices in the above sense. They could divide a dynamic component of Type B into several smaller periodic components. For convenience we include those trajectories in the set of separatrices.

\section{Language of $\mathrm{PCD}_{r 2 m}$}

In the following we assume a finite non-empty set $A$ called an alphabet, and the elements of $A$ are called letters. By $A^{*}$ we denote the set of all finite sequences of $A$ called words. A language $L$ over $A$ is a subset of $A^{*}$.

Definition 15 (Factorial language). A language $L$ over an alphabet $A$ is factorial if $u_{0} u_{1} \ldots u_{n} \in L$ implies $u_{1} u_{2} \ldots u_{n} \in L$ and $u_{0} u_{1} \ldots u_{n-1} \in L$ for arbitrary $u_{0}, \ldots, u_{n} \in A$.

Definition 16 (Prolongable language). A language $L$ over an alphabet $A$ is prolongable if for any $u \in L$ there exist $a, b \in A$ such that $a u \in L$ and $u b \in L$.

In the following we consider the words induced by the trajectories of a $\mathrm{PCD}_{r 2 m}$ in the following sense: $e_{1} \ldots e_{k}$ is a word if it is a signature of some trajectory segment.

Proposition 3. Let $\mathrm{H}=(\mathrm{Q}, \mathrm{F})$ be a $P C D_{r 2 m}$, and $L(\mathrm{H})$ be the set of all finite words generated by the trajectories of $\mathrm{H}$. Then $L(\mathrm{H})$ is a factorial and prolongable language over the alphabet $\mathrm{Bd}(\mathrm{Q})$.

Proof. By definition, $L(\mathrm{H})$ is a language over $\mathrm{Bd}(\mathrm{Q})$. It is obviously factorial and prolongable because for every boundary point $x$ there are boundary points $x_{\text {succ }}$ and $x_{\text {pred }}$ such that $x_{\text {succ }}$ is reachable from $x$ and $x$ is reachable from $x_{\text {pred }}$.

A recurrent word is an infinite word over $A$ in which every finite subword occurs infinitely often.

Definition 17 (Uniformly recurrent language). A language $L$ is called uniformly recurrent if for any $n \in \mathbb{N}$ there exists $\eta_{n} \in \mathbb{N}$ such that every word from $L$ of length $\eta_{n}$ contains all of the words from $L$ of length $n$ as subwords.

The language of a dynamic component of $\mathrm{PCD}_{r 2 m}$ can be naturally seen as a constraint of a language of $\mathrm{PCD}_{r 2 m}$ on a set of words produced by the trajectories of this component. It is a language with the same properties as in Proposition 3. The following lemma gives the sufficient condition for the language of a dynamic component of a $\mathrm{PCD}_{r 2 m}$ to be uniformly recurrent. 
Lemma 1 (Uniformity condition). Density of the trajectories of a dynamical component $C$ of $P C D_{r 2 m} \mathrm{H}=(\mathrm{Q}, \mathrm{F})$ implies the uniformal recurrence of its language $L_{C}$.

Proof. Let $w=e_{1} e_{2} \ldots e_{n}$ be a word in $L_{C}$, and $s_{w} \subseteq e_{1}$ be a maximal subinterval such that $w$ is generated by a trajectory starting at some $x \in s_{w}$.

The density of trajectories in $C$ implies that the first-return map $f: s_{w} \rightarrow s_{w}$ is defined for any $x \in s_{w}$.

To prove the uniformal recurrence of $L_{C}$ it is sufficient to show that there exists a constant $C\left(s_{w}\right)$ such that $r(x)<C\left(s_{w}\right)$ for any $x \in s_{w}$, where $r(x)$ is the length of the word corresponding to the trajectory segment connecting $x$ and $f(x)$. Then by choosing $\eta_{n}=\max _{w:|w|=n} C\left(s_{w}\right)$ we guarantee that any word of length $\eta_{n}$ contains any word of length $n$.

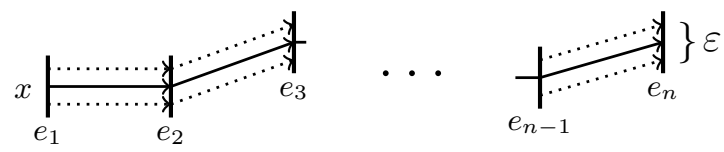

Fig. 4. " $\varepsilon$-tube" around images of $x$

We observe that for any inner point $x \in s_{w}$ there is a returning interval $i_{x} \subseteq s_{w}$ around $x$ such that $r(y)=r(x)$ for any $y \in i_{x}$. This is because a trajectory of $x$ does not meet any vertex and there is always an " $\varepsilon$-tube" around the subsequent images of $x$ (see Figure 4).

We also observe that any two adjacent returning intervals $i(x)$ and $i(y)$ are divided by a trajectory of some vertex (see Figure 5). As $C$ contains a finite number of vertices, we conclude that there is $k \in \mathbb{N}$ such that $s_{w}$ is a disjoint union of $k$ returning intervals corresponding to some $x_{1}, \ldots, x_{k} \in s_{w}$.

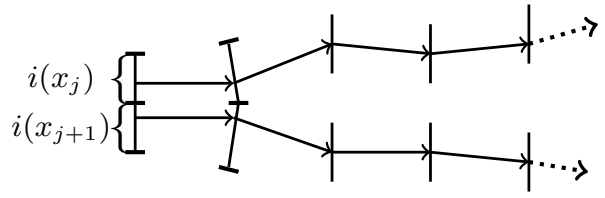

(a)

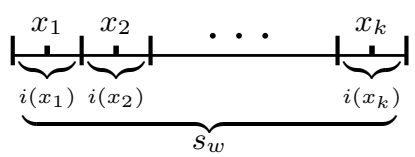

(b)

Fig. 5. An example illustrating the proof of Lemma 1

We define $C\left(s_{w}\right)=\max _{i \in\{1, \ldots, k\}} r\left(i_{i}\right)$ and this concludes the proof.

Corollary 1. The language of any dynamical component of Type B of any $P C D_{r 2 m}$ is uniformly recurrent. 


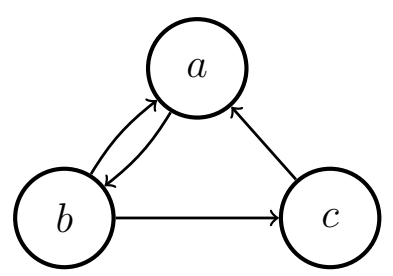

(a)

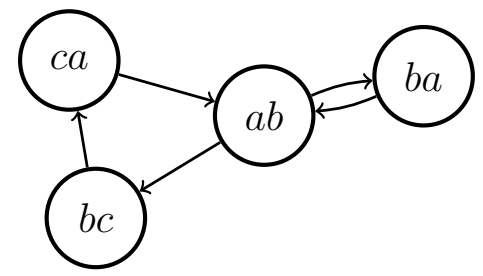

(b)

Fig. 6. (a) Rauzy graph of power 1 of the language in Example 1; (b) Rauzy graph of power 2 of the same language.

\section{Decidability of Reachability for $\mathrm{PCD}_{r 2 m}$}

In this section we show that the reachability problem is decidable for $\mathrm{PCD}_{r 2 m}$. Moreover, we provide an algorithm which decides in a finite number of steps if a target edge is reachable from an initial edge.

\subsection{Rauzy Graphs}

Definition 18 (Rauzy graph). Rauzy graph of power $k \geqslant 1$ of a language $L$ is a directed graph $R^{k}(L)=\left(V^{k}, E^{k}\right)$ defined as follows:

- $V^{k}=\{w \in L|| w \mid=k\} ;$

- For any two vertices $u=u_{1} u_{2} \ldots u_{k} \in V^{k}$ and $v=v_{1} v_{2} \ldots v_{k} \in V^{k}$ there is an edge $(u, v) \in E^{k}$ if $u_{2}=v_{1}, u_{3}=v_{2}, \ldots, u_{k}=v_{k-1}$ and $u_{1} u_{2} \ldots u_{k} v_{k} \in L$.

In other words, any two words of length $k$ are connected in the Rauzy graph of power $k$ if they are a prefix and a suffix of some word of length $k+1$.

Example 1. Let $L_{e}=\{a, b, c, a b, b c, b a, c a, a b c, a b a, b a b, b c a, c a b\}$. Then $R^{1}\left(L_{e}\right)$ and $R^{2}\left(L_{e}\right)$ will look like graphs on the Figure 6 .

\subsection{Deciding Reachability of $\mathrm{PCD}_{r 2 m}$}

In the main algorithm we will build a sequence of Rauzy graphs until the criteria, provided by the following theorem, is satisfied.

Theorem 3. Let $P C D_{r 2 m} \mathrm{H}=(\mathrm{Q}, \mathrm{F})$ have $C$ dynamic components and a language $L$. Then there exists $t_{\text {stop }} \in \mathbb{N}$ such that any $R^{i}(L), i \geqslant t_{\text {stop }}$ consists of $k \geqslant C$ disconnected components such that at least one of the following conditions holds for each component $K_{j}=\left(V_{j}, E_{j}\right)$ :

(1) All vertices in $V_{j}$ contain the same set of letters;

(2) There is $t^{\prime}<t$ such that the set of signature types of $V_{j}$ equals to the set of signature types of some $V_{j}^{\prime}$ of $R^{t^{\prime}}(L)$. 
Proof. By Theorem 2, $\mathrm{H}$ is divided into a finite number of regions with different dynamics (regions of Types A, B and C, and the separatrices forming the boundary of the regions). By Proposition 2 each separatrix starts and ends in a vertex. Let $m$ be the maximal length of a separatrix in $\mathrm{H}$. Then any $R^{i}(L)$, $i>m$ will contain at least $C$ components as there could be no words of length more than $m$ (see Figure 7).

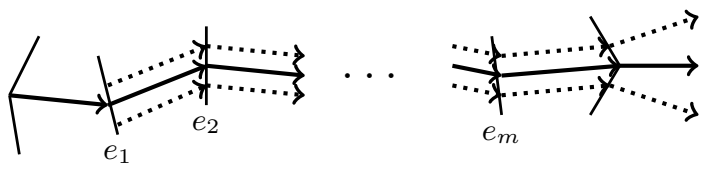

Fig. 7. An illustration of the proof of Theorem 3

Each dynamical component $D_{A}$ of Type $\mathbf{A}$ is flat and therefore can be seen as a 2-PCD. Then by Theorem $1 D_{A}$ has a finite number of signature types. All the signature types of $D_{A}$ will be constructed on some step in a sequence of Rauzy graphs and no new signature types will be later discovered. Hence, Condition (2) will eventually hold.

Each trajectory inside any component $D_{B}$ of Type B is closed and therefore periodic. It will visit the same sequence of edges, hence, Condition (1) will eventually hold.

Each trajectory inside any component $D_{C}$ of Type $\mathbf{C}$ is dense in $D_{C}$. Hence, the underlying component of a Rauzy graph is connected. By Lemma 1 the language generated by $D_{C}$ is uniformly recurrent. From this follows that there exists $\eta_{1}$ such that any word of length $\eta_{1}$ generated by any trajectory of $D_{C}$ will contain all words of length 1 (singular letters) as subwords, hence the Condition (1) will eventually hold.

Depending on which vertex belongs to which region of $\mathrm{H}$ separatrices could either generate the same words as the dynamic components or generate a finite set of words that will form a number of special components $S_{j}$ in $R^{i}(L)$. By Proposition 2 each separatrix starts and ends in a vertex and there are finite number of them, hence Condition (1) will eventually hold for each $S_{j}$.

Theorem 4. Edge-to-edge reachability for any $P C D_{r 2 m}$ is decidable.

Proof. For any $\mathrm{PCD}_{r 2 m} \mathrm{H}=(\mathrm{Q}, \mathrm{F})$, the set $V^{k}$ of vertices of $R^{k}(L)=\left(V^{k}, E^{k}\right)$ consists of all the words of length $k$ over the finite alphabet on the edges of $\mathrm{H}$. This set can be constructed by applying the successor function $k-1$ times to each edge. The edges of $R^{k}(L)$ can be computed in a finite time by checking if any two vertices have common suffix and prefix of length $k-1$.

It follows from Theorem 3 that there is a finite $t_{\text {stop }}$ such that an edge $e_{f} \in \mathrm{E}(\mathrm{Q})$ is reachable from an edge $e_{s} \in \mathrm{E}(\mathrm{Q})$ if and only if $R^{t_{s t o p}}(L)$ contains a component with a vertex labelled by a word $\left(\ldots e_{s} \ldots e_{f} \ldots\right)$. 


\section{Conclusions}

In this paper we have shown that the reachability problem for a subclass of PCDs on orientable manifolds (we called such systems Regular $\mathrm{PCD}_{2 m}$, or $\mathrm{PCD}_{r 2 m}$ ) is decidable. As future work we would be interested to extend the current results to non-orientable manifolds using properties of trajectories on non-orientable manifolds presented in [2].

\section{Acknowledgements}

The authors thank Vincent Delecroix, Alexey Kanel-Belov, Alexey Klimenko, Alexandra Skripchenko and Eugene Asarin for their kind help and consultations.

\section{References}

1. Alur, R., Courcoubetis, C., Halbwachs, N., Henzinger, T.A., Ho, P., Nicollin, X., Olivero, A., Sifakis, J., Yovine, S.: The algorithmic analysis of hybrid systems. Theoretical Computer Science 138(1), 3-34 (1995)

2. Aranson, S.H.: Trajectories on nonorientable two-dimensional manifolds. Mathematics of the USSR-Sbornik 9(3), 297-313 (1969)

3. Asarin, E., Maler, O., Pnueli, A.: Reachability analysis of dynamical systems having piecewise-constant derivatives. Theoretical Comp. Science 138(1), 35-65 (1995)

4. Asarin, E., Schneider, G.: Widening the boundary between decidable and undecidable hybrid systems. In: CONCUR 2002 - Concurrency Theory, 13th International Conference, Brno, Czech Republic, August 20-23, 2002, Proceedings. pp. 193-208 (2002)

5. Asarin, E., Schneider, G., Yovine, S.: On the decidability of the reachability problem for planar differential inclusions. In: Hybrid Systems: Computation and Control, 4th International Workshop, HSCC 2001, Rome, Italy, March 28-30, 2001, Proceedings. pp. 89-104 (2001)

6. Asarin, E., Schneider, G., Yovine, S.: Algorithmic analysis of polygonal hybrid systems, part I: Reachability. Theoretical Computer Science 379(1-2), 231-265 (2007)

7. Bell, P.C., Chen, S., Jackson, L.: On the decidability and complexity of problems for restricted hierarchical hybrid systems. Theor. Comput. Sci. 652, 47-63 (2016)

8. Bournez, O., Kurganskyy, O., Potapov, I.: Reachability problems for one-dimensional piecewise affine maps. Int. J. Found. Comput. Sci. 29(4), 529-549 (2018). https://doi.org/10.1142/S0129054118410046, https://doi.org/10.1142/S0129054118410046

9. E.Asarin, Mysore, V., Pnueli, A., Schneider, G.: Low dimensional hybrid systems - decidable, undecidable, don't know. Information and Computation 211, 138-159 (2012)

10. Henle, M.: A combinatorial introduction to topology. Dover Publications, Inc. (1979)

11. Henzinger, T.A., Kopke, P.W., Puri, A., Varaiya, P.: What's decidable about hybrid automata? Journal of Computer and System Sciences 57(1), 94-124 (August 1998) 
12. Kurganskyy, O., Potapov, I., Sancho-Caparrini, F.: Computation in onedimensional piecewise maps. In: Hybrid Systems: Computation and Control, 10th International Workshop, HSCC 2007, Pisa, Italy, April 3-5, 2007, Proceedings. pp. 706-709 (2007)

13. Laf ferriere, G., Pappas, G.J., Sastry, S.: O-m inimal hybrid systems. Mathematics of Control, Signals and Systems 13(1), 1-21 (2000)

14. Lafferriere, G., Pappas, G., Yovine, S.: A new class of decidable hybrid systems. In: Hybrid Systems: Computation and Control, Second International Workshop, HSCC'99, Berg en Dal, The Netherlands, March 29-31, 1999, Proceedings. pp. 137-151 (1999)

15. Maler, O., Pnueli, A.: Reachability analysis of planar multi-linear systems. In: CAV. pp. 194-209 (1993)

16. Mayer, A.: Trajectories on the closed orientable surfaces. Rec. Math. [Mat. Sbornik] N.S. 12(54):1, 71-84 (1943)

17. Mysore, V., Pnueli, A.: Refining the undecidability frontier of hybrid automata. In: FSTTCS 2005: Foundations of Software Technology and Theoretical Computer Science, 25th International Conference, Hyderabad, India, December 15-18, 2005, Proceedings. pp. 261-272 (2005)

18. Rauzy, G.: Suites à termes dans un alphabet fini. In: Seminar on number theory (1982-1983), University of Bordeaux I, Talence, 25. pp. 1 - 16 (1983) 